\title{
ESGAR consensus statement on liver MR imaging and clinical use of liver-specific contrast agents
}

\author{
E. Neri ${ }^{1}$ - M. A. Bali ${ }^{2}$ - A. Ba-Ssalamah ${ }^{3} \cdot$ P. Boraschi $^{1} \cdot$ G. Brancatelli $^{4}$ • \\ F. Caseiro Alves ${ }^{5}$ - L. Grazioli ${ }^{6}$ - T. Helmberger ${ }^{7}$ - J. M. Lee $^{8}$ • R. Manfredi $^{9}$ • \\ L. Martì-Bonmati ${ }^{10}$ - C. Matos $^{2}$ - E. M. Merkle ${ }^{11}$ - B. Op De Beeck ${ }^{12}$ - W. Schima ${ }^{13}$. \\ S. Skehan ${ }^{14} \cdot$ V. Vilgrain ${ }^{15} \cdot$ C. Zech $^{16} \cdot$ C. Bartolozzi ${ }^{1}$
}

Received: 30 December 2014 /Revised: 7 June 2015 / Accepted: 22 June 2015 / Published online: 21 July 2015

(C) The Author(s) 2015. This article is published with open access at Springerlink.com

\begin{abstract}
Objectives To develop a consensus and provide updated recommendations on liver MR imaging and the clinical use of liver-specific contrast agents.

Methods The European Society of Gastrointestinal and Abdominal Radiology (ESGAR) formed a multinational European panel of experts, selected on the basis of a literature review and their leadership in the field of liver MR imaging. A modified Delphi process was adopted to draft a list of statements. Descriptive and Cronbach's statistics were used to rate levels of agreement and internal reliability of the consensus.
\end{abstract}

\section{E. Neri}

emanuele.neri@med.unipi.it

1 Department of Diagnostic and Interventional Radiology, University of Pisa, Pisa, Italy

2 Department of Radiology, Hôpital Erasme, MRI Clinics, Bruxelles, Belgium

3 Department of Biomedical Imaging and Image-guided Therapy, The General Hospital of the Medical University of Vienna, Vienna, Austria

4 Department of Radiology, University of Palermo, Palermo, Italy

5 Medical Imaging Department and Faculty of Medicine, University Hospital of Coimbra, Coimbra, Portugal

6 Department of Radiology, Spedali Civili di Brescia, Brescia, Italy

7 Department of Diagnostic and Interventional Radiology and Nuclear Medicine, Klinikum Bogenhausen, Academic Teaching Hospital of the Technical University, Munich, Germany

Results Three Delphi rounds were conducted and 76 statements composed on MR technique ( $\mathrm{n}=17)$, clinical application of liver-specific contrast agents in benign, focal liver lesions $(n=7)$, malignant liver lesions in non-cirrhotic $(n=9)$ and in cirrhotic patients $(\mathrm{n}=18)$, diffuse and vascular liver diseases $(n=12)$, and bile ducts $(n=13)$. The overall mean score of agreement was 4.84 (SD \pm 0.17 ). Full consensus was reached in $22 \%$ of all statements in all working groups, with no full consensus reached on diffuse and vascular diseases.

Conclusions The consensus provided updated recommendations on the methodology, and clinical indications, of MRI National University College of Medicine, Seoul, Korea

9 Department of Radiology, University of Verona, Verona, Italy

10 Área Clínica de Imagen Médica, Hospital Universitario y Politécnico La Fe, Valencia, Spain

11 Klinik für Radiologie und Nuklearmedizin, Universitätsspital Basel, Basel, Switzerland

12 Department of Radiology, Antwerp University Hospital, Edegem, Belgium

13 Department of Diagnostic and Interventional Radiology, KH Goettlicher Heiland, Krankenhaus der Barmherzigen Schwestern and Sankt Josef-Krankenhaus, Vienna, Austria

14 Department of Radiology, St Vincent's University Hospital, Elm Park, Dublin, Ireland

15 Radiology Department, Assistance Publique-Hôpitaux de Paris, APHP, Hôpital Beaujon, Clichy Paris, France

16 Abteilungsleiter Interventionelle Radiologie, Klinik für Radiologie und Nuklearmedizin, Universitätsspital Basel, Basel, Switzerland 
with liver specific contrast agents in the study of liver diseases.

Key points

- Liver-specific contrast agents are recommended in MRI of the liver.

- The hepatobiliary phase improves the detection and characterization of hepatocellular lesions.

- Liver-specific contrast agents can improve the detection of HCC.

Keywords Liver - Biliary tract - Magnetic resonance imaging $\cdot$ Contrast media $\cdot$ Delphi technique

\section{Introduction}

The advantages of MR imaging in the investigation of the liver are well documented since this examination provides a comprehensive work-up of focal and diffuse liver diseases. Recent state-of-the-art techniques including fast scanning acquisitions and new MR imaging contrast agents enable improvements in detection and characterization of focal liver lesions. Therefore, together with appropriate clinical information, in most cases, a definitive diagnosis can be adequately achieved avoiding invasive procedures such as liver biopsy. This is based on the unique properties of MR imaging resulting in a high intrinsic soft tissue contrast between normal liver parenchyma and liver lesions, which can be further enhanced with intravenous administration of non-specific (extracellular) and liver-specific (hepatobiliary) gadoliniumbased contrast agents [1-4].

Multiphasic dynamic gadolinium-enhanced imaging, which is considered essential in detection and characterization of liver lesions, is routinely obtained by using non-specific intravenous contrast agents that distribute in the extracellular space, both within and outside the vessels, and have imaging dynamics comparable to the extracellular iodinated contrast media used in CT [5, 6].

The so-called liver-specific (or hepatobiliary) contrast agents (gadobenate dimeglumine, Gd-BOPTA, and gadoxetic acid, Gd-EOB-DTPA), are characterized by a dual behaviour: by exhibiting elimination through both renal and hepatic excretion pathways and thereby possessing both early perfusion information (renal elimination pathway) and, later, hepatocyte-selective information (hepatic excretion pathway) mediated through protein transporters, located in the canalicular or sinusoidal pole of the hepatocytes [7-9].

The liver-specific contrast agents are Gd-based compounds and, therefore, shorten the $\mathrm{T} 1$ relaxation time that results in an increased signal intensity of the healthy liver parenchyma on T1-weighted images $[8,10]$.

The clinical use of liver-specific contrast agents allows the physician to obtain morphologic and vascular-related information, thanks to the dynamic study, as well as functional information, thanks to the hepatocyte-selective phase of enhancement. However, even if a large number of research and review articles on the use of liver-specific contrast agents in different clinical scenarios have already been published, agreement about their clinical indication is still lacking [11-16].

To develop a consensus and provide updated recommendations on the best MRI technique and the clinical use of liverspecific contrast agents, the European Society of Gastrointestinal and Abdominal Radiology (ESGAR) recruited a working group of key opinion leaders in the field of liver MR imaging.

\section{Materials and method}

\section{Consensus panel}

The working group, composed of a multinational European panel of 18 members and faculty of the ESGAR (composed of X.X., ${ }^{1}$ B.M.A., M.A., B.P., B.G., CA.F., G.F., H.T., L.J.M., M.R., MB.L., M.C., M.EM., ODB.B., S.W., S.S., V.V., Z.C.), used a modified Delphi process to rate the level of agreement on numerous statements pertaining to the MR imaging technique and the clinical applications of liver-specific contrast agents [17]. Two additional panellists, who did not express a vote, were chosen to play the role of facilitators (C.B. and E.N.).

Three Delphi rounds were conducted. In the first round, the panellists had a face-to-face meeting and, on the basis of their main area of research and expertise, were split into six working groups (WG) on MR technique (WG 1), benign focal liver lesions (WG 2), malignant liver lesions in non-cirrhotic patients (WG 3), focal liver lesions in cirrhotic patients (WG 4), diffuse and vascular liver diseases (WG 5), and bile duct applications (WG 6). Each WG independently drafted a cluster of statements pertaining to their allocated subject. A preliminary literature review for each WG to support the composition of statements was based on the GRADE system [18]. Each WG then presented the proposed statements to the whole panel for detailed discussion. At this time, the content and wording of statements were modified until a general consensus emerged.

In the second and third rounds the panellists were sent electronic copies of the latest statements in order to rate independently their level of agreement using a 5-point Likert scale as follows: 1 , strongly disagree with the statement; 2 , disagree somewhat with the statement; 3 , undecided; 4 , agree somewhat with the statement; 5 , strongly agree with the statement. To reach the maximum consensus, the number and content of

\footnotetext{
${ }^{1}$ X.X. refers to a panelist who left the panel after the third round.
} 
statements was modified in the iteration from the second to the third round.

\section{Statistical analysis}

All ratings of panellists for each statement were analysed with descriptive statistics measuring the mean score, the maximum and minimum score, and the standard deviation.

A mean score of 4 was considered a good agreement between panellists and a score of 5 a complete agreement.

After the second round, the facilitator collected the ratings from the panellists and calculated the score of agreement for each statement. If the mean score was less than 4 , the facilitator asked the panellists to review the statement and reach a higher level of agreement by changing the content and, when necessary, the number of the statements.

To measure the internal consistency of the panellists ratings for each cluster of statements, a quality analysis based on the average inter-item correlation was performed with Cronbach's alpha $(\mathrm{C} \alpha)$ correlation coefficient, using SPSS (SPSS, Chicago, IL, USA) $[19,20]$. The $\mathrm{C} \alpha$ test provides a measure of the internal consistency of a test or scale; it is expressed as a number between 0 and 1 . Internal consistency describes the extent to which all the items in a test measure the same concept. $\mathrm{C} \alpha$ was determined after each round.

The closer $\mathrm{C} \alpha$ coefficient is to 1.0, the greater the internal consistency of the items in the scale. An alpha coefficient $(\alpha)>0.9$ was considered excellent, $\alpha>0.8$ Good, $\alpha>0.7$ Acceptable, $\alpha>0.6$ Questionable, $\alpha>0.5$ Poor, and $\alpha<0.5$ Unacceptable. However, in the iterations a $\alpha$ of 0.8 was considered a reasonable goal for internal reliability.

\section{Results}

In the second round, the panel elaborated 94 statements. These were reduced to 76 in the third round (Table 1). The overall mean score of agreement of the experts was 4.72 (standard deviation $\mathrm{SD} \pm 0.22$ ) in the second round and improved to 4.84 (SD \pm 0.17 ) in the third round. From the second to the third round, the panel reached complete consensus (rating 5), respectively, in 12/93 (12\%) and 17/76 (22\%) statements. Meanwhile, in the remaining statements, in both rounds, no expert rated any individual statement less than 4, confirming, despite the apparent heterogeneity of the sample, that there was generally some agreement amongst them.

Full consensus was reached by the experts panel in $22 \%$ of the statements. In the remaining statements, full consensus was not reached, but all the panellists achieved a "good" level of agreement.

The highest mean level of agreement $(4.87 \pm 0.21)$ and questionable internal consistency $(\mathrm{C} \alpha 0.67)$ was reached by the WG1 (MR technique), with full consensus on the statements about the use of MR coils, type of contrast agent, and the specific MR sequences to be used in liver MR examinations.

The highest number of statements $(6 / 18 ; 33 \%)$ having full consensus were in the WG4, with a good mean level of agreement $(4.85 \pm 0.15)$ and questionable internal consistency $(\mathrm{C} \alpha$ $0.61)$. Such statements clearly addressed the proposed "stateof-the-art" MR protocol and the enhancement pattern observed with liver-specific contrast agents of focal liver lesions in cirrhotic patients.

\section{Discussion}

Along the entire consensus process, the experts completed three rounds; the first round served to elaborate the basic statements, whereas the second and third rounds contained the core of the discussion and were necessary to reach the maximum consensus, in order to create an optimized and homogeneous opinion for each statement. Finally, the overall mean score of the panellists was 4.84 ( $\mathrm{SD} \pm 0.17$ ), which should be considered an almost excellent result of agreement.

The Cronbach's test used in the study had the value of an additional measure of consistency of the consensus rounds; the highest $\mathrm{C} \alpha$ were obtained in the $\mathrm{Wg} 1$ and WG4, but with a questionable internal consistency $(0.6<\mathrm{C} \alpha<0.7)$. In the remaining WGs the $\mathrm{C} \alpha$ was below an acceptable level. These results do not imply unreliability of the consensus; in fact, from a practical point of view, such results are related to the high number of panellists $(n=18)$ that inevitably raised the variability in the score of agreement. However, such heterogeneity of scores (all higher than 4) did not significantly impact the mean level of agreement. Moreover, this reflects the mediating role played by the two facilitators of the consensus that allowed pushing the levels of agreement above the threshold of 4 .

All panellists exhibited a high level of agreement for the MR technique with clear recommendations regarding the use of MR coils, type of contrast agent, and the specific MR sequences to be used in liver MR examinations. These data reflect a consolidated approach to liver MR examination with no significant difference among panel members despite their wide geographical spread. All panel members, belonging to academic and non-academic centres, are regular speakers of ESGAR meetings/workshops, a condition that may have facilitated the information sharing in this field of expertise and, therefore, improved their level of agreement.

However, even if this condition may have facilitated the agreement, the recruitment of experts and specialist belonging to the same peer group does not necessarily compromise expertise. But it has to be taken into account that there is a risk of mutual influencing of opinions and attitudes. A way to overcome this bias would have been to include panel members 


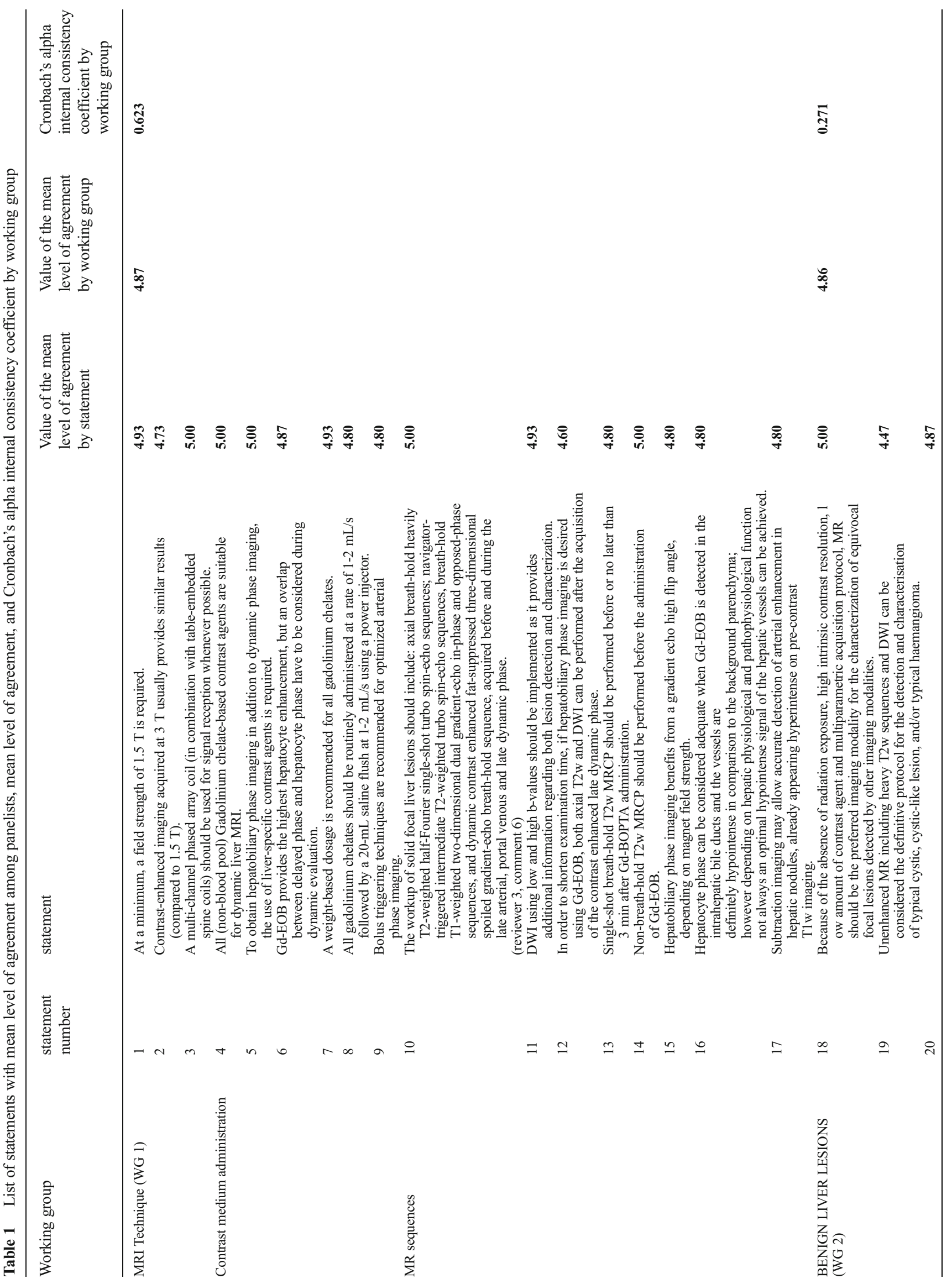




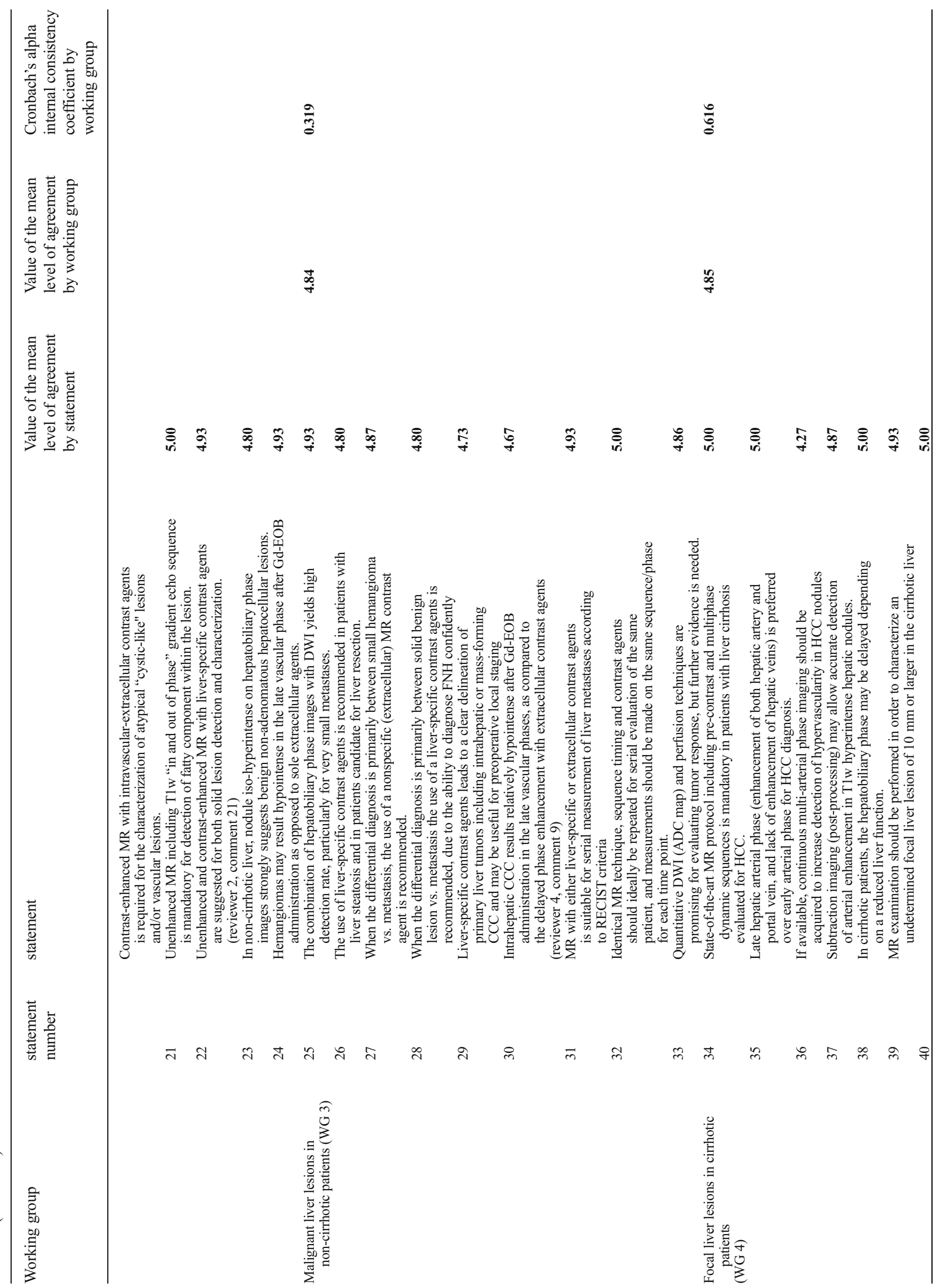




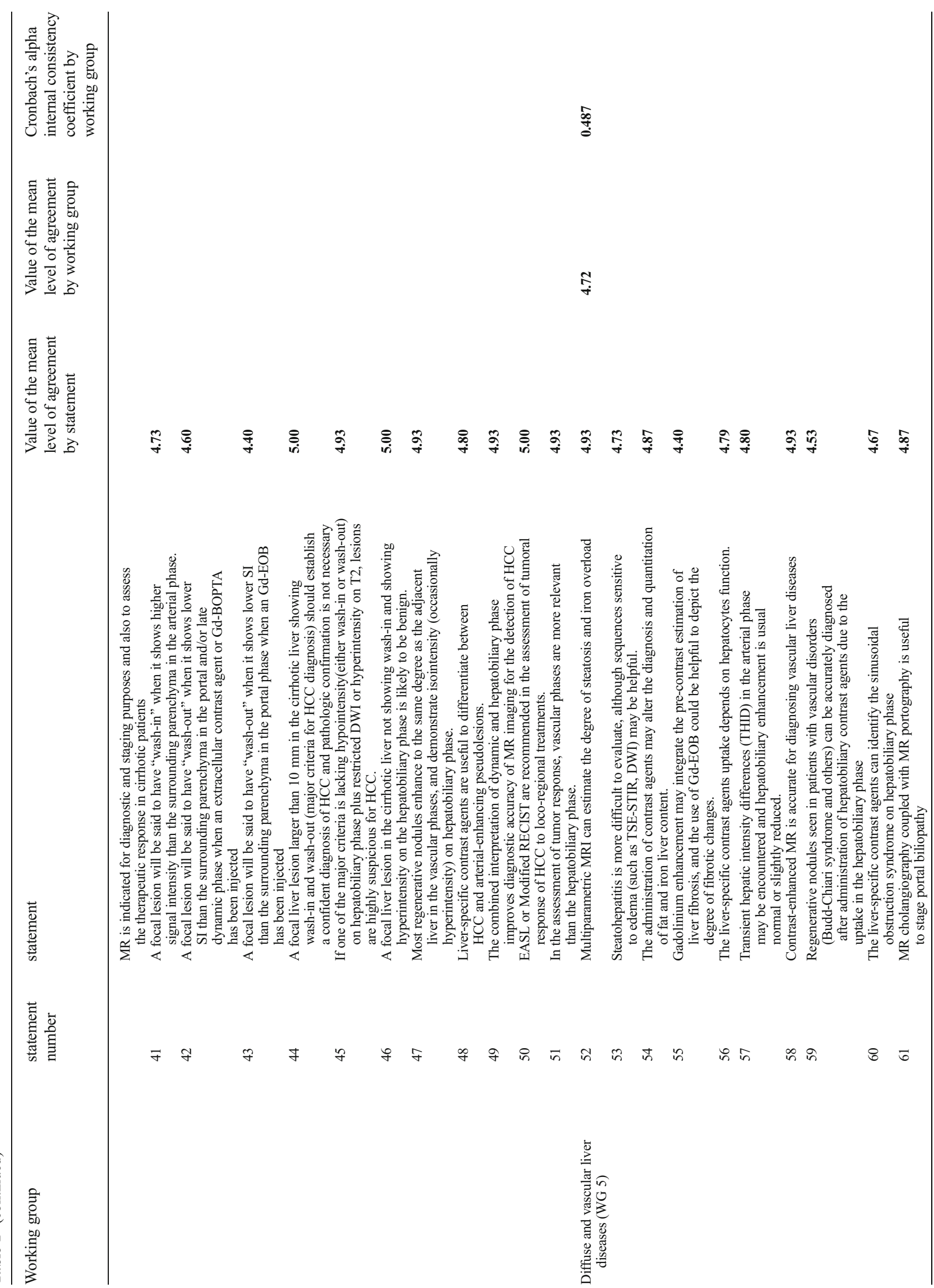




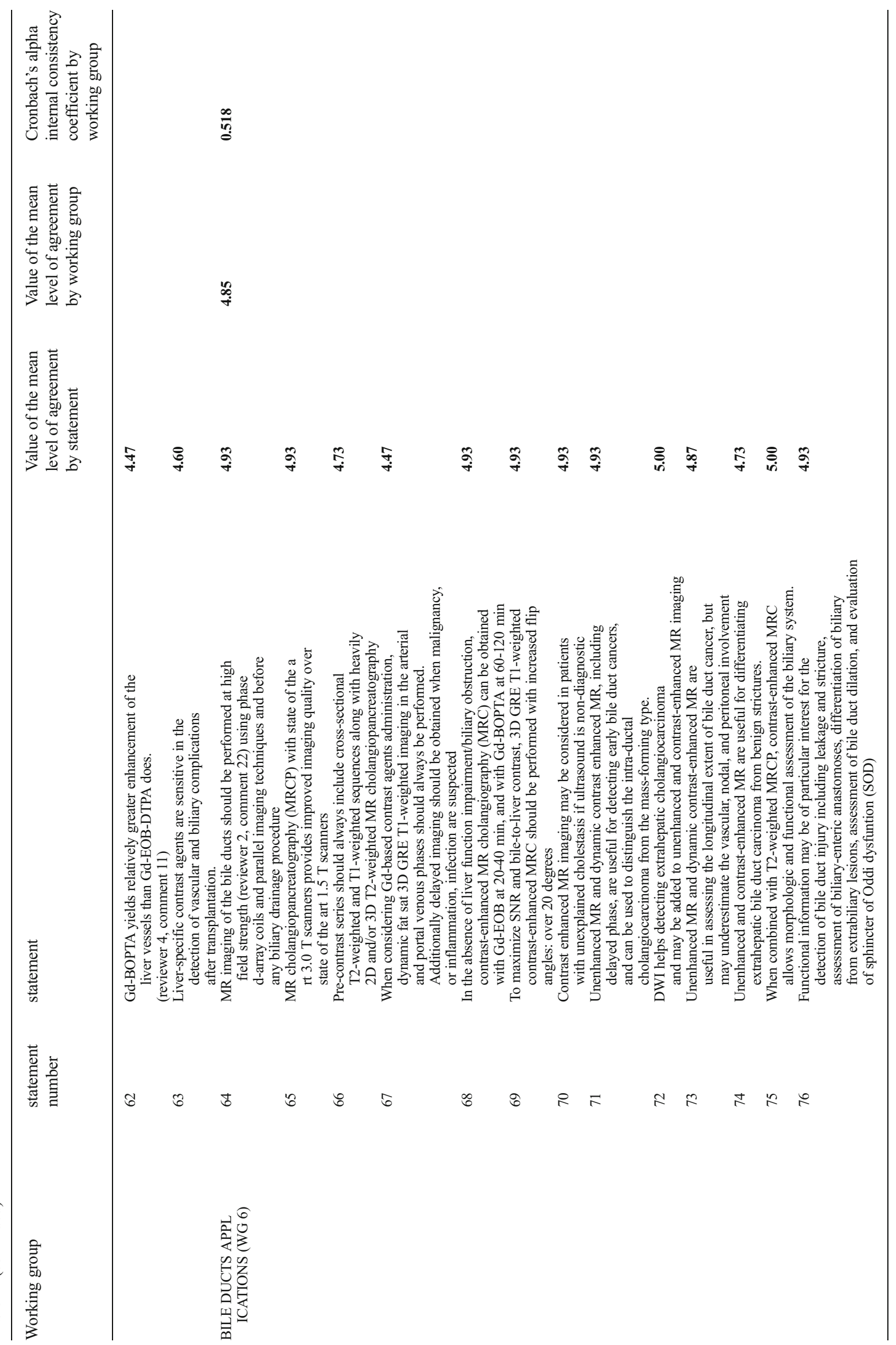


with expertise in other specialized areas such as hepatology, surgery, and methodology, and a representation from sister medical societies.

As a basic rule of MR technique, all the panellists clearly addressed that the workup of solid focal liver lesions should include axial T2- and T1-weighted sequences, followed by T1-weighted gradient dual echo images, DWI (using low and high b-values) and dynamic contrast-enhanced T1weighted fat-saturated (see statement 10 in Table 1). However, no full consensus was reached on statements 1 and 2 that addressed similar results of MR imaging at 3 and $1.5 \mathrm{~T}$. This incomplete agreement can be explained by the discrepancies of the comparative studies on the use of 1.5 vs. 3 T MRI [21-24].

The remaining statements regarding MRI technique, even with less than "full" consensus, definitively addressed the modalities of contrast medium administration (flow-rate of $1-2 \mathrm{~mL} / \mathrm{s}$ followed by a $20-\mathrm{mL}$ saline flush at $1-2 \mathrm{~mL} / \mathrm{s}$ using a bolus triggering technique ) and timing of T2-weighted and DWI sequences (after the acquisition of the contrast-enhanced late dynamic phase).

With regards to the recommendations on the use of the gadobenate dimeglumine (Gd-BOPTA) and the gadoxetic acid (Gd-EOB-DTPA) that are the currently available on the market, the panel was aware there are no data indicating diagnostic superiority of one agent over the other. It must also be noted that to our knowledge there are very few publications comparing Gd-EOB-DTPA and Gd-BOPTA [25-27].

All panellists fully agreed that all non-blood pool gadolinium chelate-based contrast agents are suitable for dynamic liver MRI, but the use of liver-specific contrast agents is mandatory to obtain the hepatobiliary phase in addition to the dynamic phase (statements 4 and 5). Jeong et al emphasized that the "hepatobiliary" phase is where uptake by the hepatocytes and excretion to the bile ductule have reached an optimal level for diagnosis [28]. Kim et al demonstrated no statistically significant difference in the signal intensity of liver parenchy$\mathrm{ma}$ in the arterial phase between gadobenate dimeglumine and gadoxetic acid [28], and Filippone et al showed a statistically significant higher signal intensity of gadoxetic acid-enhanced MR in the hepatobiliary phase [29]. In a recent article, BaSsalamah et al stated that liver specific contrast agents provide a multiparametric assessment of hepatobiliary diseases, since they combine morphologic and functional information in the same setting and, therefore, can play the role of imaging biomarkers [30].

A mean good level of agreement was reached between the panellists regarding the application of liver specific contrast agents in benign hepatocellular liver lesions, and addressed MRI as the preferred imaging modality for the characterization of equivocal focal lesions detected by other imaging modalities (statement 18). The added value of Gd-BOPTA in the evaluation of solid hepatic lesions is supported by literature evidence. Grazioli et al, through a quantitative analysis of signal intensity, lesion-to-liver contrast, and enhancement ratio, demonstrated that Gadoxetic acid-enhanced MR imaging facilitates the differential diagnosis of hepatocellular adenoma (HCA) and focal nodular hyperplasia (FNH) [31]. The same author showed in a previous article that this was possible with Gd-BOPTA [32]. Haimerl et al and Morana et al showed that the hepatobiliary phase images obtained after GdEOB-DTPA-enhanced dynamic MRI significantly improved the index of diagnostic performance in the differentiation of focal solid lesions [33, 34].

A mean good level of agreement was also reached between the panellists on the cluster of statements about malignant liver lesions in non-cirrhotic patients, where the use of liver-specific contrast agents has been recommended to improve the differential diagnosis between a solid benign hepatocellular lesion and metastasis, and delineation of primary liver tumors (including intrahepatic or mass-forming cholangiocarcinoma). In a recent study comparing the diagnostic performance of MDCT and gadoxetic acid-enhanced MRI at 3.0, Scharitzer et al found that MRI with the liverspecific contrast agent had better performance in the assessment of small colorectal liver metastases [35]. A similar study comparing MDCT and MRI performed by Kim et al, showed higher diagnostic accuracy for MRI in the detection of hepatic metastases and for the differential diagnosis with hepatic haemangiomas or cysts [36]. However, a hypointense pattern in the hepatobiliary phase has been reported by Han et al [37] as a potential pitfall of liver-specific contrast agents agents in patients who received oxaliplatin as part of their chemotherapy regimen. Specifically, in these patients, due to the sinusoidal obstruction syndrome (focal hepatopathy), the morphologic pattern of the hypointensity at the hepatobiliary phase may contribute to avoid this potential pitfall.

With regard to mass-forming cholangiocarcinoma, the panel clearly stated that concerning the delayed phase enhancement obtained with non-specific extracellular agents, GdEOB provides a relative hypointense MRI pattern of the lesion both in the transitional and hepatobiliary phases that improves tumour conspicuity [38]. The panel suggested also the use of DWI and perfusions techniques, and recently, Park et al demonstrated that the target appearance seen on the DWI was the most reliable imaging feature for distinguishing small massforming peripheral cholangiocarcinoma from small HCC [39].

The best agreement among the panellists was reached for focal liver lesions in cirrhotic patients. The panel stated with full agreement that a confident diagnosis of HCC by using a complete dynamic study with pre-contrast and multiphase sequences (statement 34), can be optimally reached with a late hepatic arterial phase over early arterial phase (statement 35), and the hepatobiliary phase may be delayed depending on a reduced liver function (statement 38) [40, 41]. Haimerl et al demonstrated that regarding comparability with the 
MELD (Model for End-Stage Liver Disease) score, decreased Gd-EOB-DTPA accumulation in the hepatocytes during the hepatobiliary phase, and $\mathrm{T} 1$ relaxometry correlated with reduced liver function [42]. Verloh et al also found that the relative enhancement during hepatobiliary phase in GDEOB-DTPA MRI correlates with the MELD score [43].

Of note, the panellists addressed that the use of liverspecific contrast agents has particular usefulness in improving the detection of HCC. In fact, while statements 41, 42, and 43 clearly address the definition of wash-in and wash-out (higher signal intensity than the surrounding parenchyma in arterial phase, lower signal intensity in the portal phase after injection of Gd-chelates), statement 48 addresses the importance of the hepatobiliary phase to differentiate between HCC and arterialenhancing pseudolesion when using liver specific contrast media.

Cereser et al have shown that the delayed phase is superior to the portal venous phase for the wash-out detection in hypervascular HCC with Gd-BOPTA MRI in the cirrhotic liver. This is one of the limitations of Gd-EOB-DTPA, where the hypointensity on the 3-5 min delayed phase, also called the transitional phase, is less effective in detecting washout as it blends into the liver specific phase. Accordingly, often wash-out cannot be assessed with Gd-EOB-DTPA [44].

In a study performed by Bartolozzi et al the hyperintensity on the arterial phase and hypointensity on the transitional phase were highly predictive for $\mathrm{HCC}$, but a further element of HCC diagnosis was the hypointensity on hepatobiliary phase, evident in 39 out of 40 the HCC detected in the study cohort with a $100 \%$ positive predictive value in suggesting nodular premalignancy/malignancy [45]. Yu et al supported the same statement, and has since demonstrated that even the detection of small HCC nodule $(<1 \mathrm{~cm})$ in a standard multiphasic study can be improved by the addition of the hypointensity pattern on the hepatobiliary phase [46]. Granito et al found that hypointensity during the MR hepatobiliary phase was observed in all HCC nodules of the study and concluded that gadoxetic acid MRI may enhance the sensitivity of the non-invasive diagnosis of small hepatocellular carcinoma nodules in cirrhotic patients under surveillance [47]. In summary, the panel suggests that in cirrhotic patients, the hepatic arterial phase and portal venous phase might not be sufficient to establish a confident diagnosis of HCC and should be integrated by the hepatobiliary phase.

No statement reached full agreement for diffuse and vascular liver diseases, and it was acknowledged that a correct estimate of the degree of steatosis and iron overload needs multiparametric MRI, even if the administration of contrast agents may alter the quantification of fat and iron liver content. Even with the support of the few literature studies available, the use of liver-specific contrast agents was specifically indicated to evaluate multiacinar regenerative nodules in patients with vascular disorders (statement 59) in the study of the sinusoidal obstruction syndrome (statement 60) and in the detection of vascular and biliary complications after transplantation (statement 63). In a study by Katajiama et al, the hepatic congestion and oedema occurring in Budd-Chiari syndrome were seen as slightly hypointense areas on Gd-EOB-DTPAenhanced hepatobiliary-phase images, and such a pattern improved the diagnosis of sinusoidal obstruction syndrome with respect to Gd-DTPA enhancement [48]. In a recent study, Shin et al confirmed that Gd-EOB provides a peculiar reticular hypointensity pattern on hepatobiliary phase images and that is highly specific for the diagnosis of sinusoidal obstruction syndrome in patients with treated colorectal hepatic metastases [49].

The final cluster of statements indicates that the evaluation of the biliary tract should be an integrant part of the liver study, and MRCP should be performed on pre-contrast series with heavily 2D and/or 3D T2-weighted sequences. It should be taken into account that in the absence of liver function impairment/biliary obstruction, contrast-enhanced MR cholangiography (MRC) can be optimally obtained with GdEOB-DTPA at 20 min after injection (statements 66 and 68). Hepatic excretion of liver-specific contrast agents results in enhancement of biliary structures, and it is likely to have a great impact on better visualization of biliary system. On the basis of these characteristics, it may potentially increase reliability of the MR examination or decrease the occurrence of a non-diagnostic or equivocal interpretation [50]. This emerging diagnostic tool, especially when using Gd-EOB-DTPA, is particularly helpful for delineating the anatomy of the biliary tract and detecting post-operative complications such as anastomotic and non-anastomotic strictures and biliary leaks. In addition, it can provide functional information that is extremely promising in the grading of biliary obstruction. Recently, Boraschi et al in review papers showed the usefulness of Gd-EOB in the evaluation of biliary-enteric anastomoses and in the assessment of biliary complications after orthotopic liver transplantation [51, 52]. The drawbacks of contrastenhanced MRC include its high cost (it is also a timeconsuming technique) and limitations in depicting the biliary system in patients with hepatobiliary dysfunction.

\section{Conclusion}

The panel of the ESGAR working group covered most important aspects of liver MRI methodology combined with the clinical use of liver-specific contrast agents, and reached a good level of agreement on most statements. As a result of the consensus process, the working group provided updated recommendations on the best use of MRI in the study of liver diseases.

Such recommendations should be helpful for both the radiologist who is starting MR imaging of the liver and for those 
who have already applied the technique, but whose practice may need updating in the light of more recent developments.

Acknowledgments The scientific guarantor of this publication is Carlo Bartolozzi. The authors of this manuscript declare relationships with the following companies:

Jeong Min Lee received grants from Donseo Medical, CMS, Acuzen, Starmed Ltd., RF MEDICAL, Bayer Healthcare outside the submitted work.

Elmar Merkle received grants from Siemens, Bracco, Bayer, Guerbet outside the submitted work and is a member of the Scientific Advisory Board of Siemens and Bayer, and lecturer for Siemens and Bayer. Ahmed $\mathrm{Ba}-\mathrm{Ssalamah}$ is a lecturer from Bayer Healthcare, Siemens and Sanochemia. Wolfgang Schima is a lecturer for Siemens.

The authors state that this work has not received any funding. No complex statistical methods were necessary for this paper. Institutional Review Board approval and written informed consent was not required because this is a consensus paper. The work is part of a series of guidelines drawn up by ESGAR and previously published in European Radiology. In particular, the study was performed using the same methodology of the Neri, Halligan, Hellstrom et al (2013) 2nd ESGAR consensus statement on CT Colonography. Eur Radiol 23 (3): 720-9.

Methodology: Retrospective analysis of literature

Open Access This article is distributed under the terms of the Creative Commons Attribution-NonCommercial 4.0 International License (http:// creativecommons.org/licenses/by-nc/4.0/), which permits any noncommercial use, distribution, and reproduction in any medium, provided you give appropriate credit to the original author(s) and the source, provide a link to the Creative Commons license, and indicate if changes were made.

\section{References}

1. Bellin MF, Vasile M, Morel-Precetti S (2003) Currently used nonspecific extracellular MR contrast media. Eur Radiol 13:2688-2698

2. Balci NC, Semelka RC (2005) Contrast agents for MR imaging of the liver. Radiol Clin N Am 43:887-898

3. Erturk SM, Alberich-Bayarri A, Herrmann KA, Marti-Bonmati L, Ros PR (2009) Use of 3.0-T MR imaging for evaluation of the abdomen. Radiographics 29:1547-1563

4. Kudo M (2011) Diagnostic imaging of hepatocellular carcinoma: recent progress. Oncology 81:73-85

5. Zech CJ, Bartolozzi C, Bioulac-Sage P et al (2013) Consensus report of the fifth international forum for liver MRI. AJR Am J Roentgenol 201:97-107

6. Choi JY, Lee JM, Sirlin CB (2014) CT and MR imaging diagnosis and staging of hepatocellular carcinoma: part I. Development, growth, and spread: key pathologic and imaging aspects. Radiology 272:635-654, reviewer 2, comment 24

7. van Montfoort JE, Stieger B, Meijer DK, Weinmann HJ, Meier PJ, Fattinger KE (1999) Hepatic uptake of the magnetic resonance imaging contrast agent gadoxetate by the organic anion transporting polypeptide Oatp1. J Pharmacol Exp Ther 290:153-157

8. Seale MK, Catalano OA, Saini S, Hahn PF, Sahani DV (2009) Hepatobiliary-specific MR contrast agents: role in imaging the liver and biliary tree. Radiographics 29:1725-1748

9. Semelka RC, Helmberger TK (2001) Contrast agents for MR imaging of the liver. Radiology 218:27-38

10. Ringe KI, Husarik DB, Sirlin CB, Merkle EM (2010) Gadoxetate disodium-enhanced MRI of the liver: Part 1, protocol optimization and lesion appearance in the noncirrhotic liver. AJR 195:13-28
11. Goodwin MD, Dobson JE, Sirlin CB, Lim BG, Stella DL (2011) Diagnostic challenges and pitfalls in MR imaging with hepatocytespecific contrast agents. RadioGraphics 31:1547-1568

12. Van Beers BE, Pastor CM, Hussain HK (2012) Primovist, Eovist: what to expect? J Hepatol 57:421-429

13. Guglielmo FF, Mitchell DG, Gupta S (2014) Gadolinium contrast agent selection and optimal use for body MR imaging. Radiol Clin N Am 52:637-656

14. Ronot M, Vilgrain V (2014) Hepatocellular carcinoma: diagnostic criteria by imaging techniques. Best Pract Res Clin Gastroenterol 28:795-812

15. Cogley JR, Miller FH (2014) MR imaging of benign focal liver lesions. Radiol Clin N Am 52:657-682

16. Hope TA, Ohliger MA, Qayyum A (2014) MR imaging of diffuse liver disease: from technique to diagnosis. Radiol Clin N Am 52: 709-724

17. Graham B, Regehr G, Wright JG (2003) Delphi as a method to establish consensus for diagnostic criteria. J Clin Epidemiol 56: $1150-1156$

18. Guyatt G, Oxman AD, Akl EA et al (2011) GRADE guidelines: 1. Introduction-GRADE evidence profiles and summary of findings tables. J Clin Epidemiol 64:383-394

19. Bravo G, Potvin L (1991) Estimating the reliability of continuous measures with Cronbach's alpha or the intraclass correlation coefficient: toward the integration of two traditions. J Clin Epidemiol 44: 381-390

20. Cronbach L (1951) Coefficient alpha and the internal structure of tests. Psychometrika 16:3

21. Ramalho M, Altun E, Herédia V, Zapparoli M, Semelka R (2007) Liver MR imaging: $1.5 \mathrm{~T}$ versus 3T. Magn Reson Imaging Clin $\mathrm{N}$ Am 15:321-347

22. Merkle EM, Dale BM, Paulson EK (2006) Abdominal MR imaging at 3T. Magn Reson Imaging Clin N Am 14:17-26

23. von Falkenhausen MM, Lutterbey G, Morakkabati-Spitz $\mathrm{N}$ et al (2006) High-field-strength MR imaging of the liver at 3.0 T: intraindividual comparative study with MR imaging at $1.5 \mathrm{~T}$. Radiology 241:156-166 (reviewer 2, comment 15)

24. Chang JM, Lee JM, Lee MW et al (2006) Superparamagnetic iron oxide-enhanced liver magnetic resonance imaging: comparison of $1.5 \mathrm{~T}$ and $3.0 \mathrm{~T}$ imaging for detection of focal malignant liver lesions. Investig Radiol 41:168-174 (reviewer 2, comment 15)

25. Gupta RT, Iseman CM, Leyendecker JR, Shyknevsky I, Merkle EM, Taouli B (2012) Diagnosis of focal nodular hyperplasia with MRI: multicenter retrospective study comparing gadobenate dimeglumine to gadoxetate disodium. AJR Am J Roentgenol 199: 35-43 (reviewer 4, comment 2)

26. Karam AR, Shankar S, Surapaneni P, Kim YH, Hussain S (2010) Focal nodular hyperplasia: central scar enhancement pattern using gadoxetate disodium. J Magn Reson Imaging 32:341-344 (reviewer 4, comment 2)

27. Park Y, Kim SH, Kim SH et al (2010) Gadoxetic acid (Gd-EOBDTPA)-enhanced MRI versus gadobenate dimeglumine (GdBOPTA)-enhanced MRI for preoperatively detecting hepatocellular carcinoma: an initial experience. Korean J Radiol 11:433-440 (reviewer 4, comment 2)

28. Jeong WK, Kim YK, Song KD, Choi D, Lim HK (2013) The MR imaging diagnosis of liver diseases using gadoxetic acid: emphasis on hepatobiliary phase. Clin Mol Hepatol 19:360-366 (reviewer 2, comment 24)

29. Kim HJ, Kim BS, Kim MJ et al (2013) Enhancement of the liver and pancreas in the hepatic arterial dominant phase: comparison of hepatocyte-specific MRI contrast agents, gadoxetic acid and gadobenate. Reson Imaging 37:903-938 (reviewer 2, comment 24)

30. Ba-Ssalamah A, Qayyum A, Bastati N, Fakhrai N, Herold CJ, Caseiro Alves F (2014) P4 radiology of hepatobiliary diseases with 
gadoxetic acid-enhanced MRI as a biomarker. Expert Rev Gastroenterol Hepatol 8:147-160

31. Grazioli L, Bondioni MP, Haradome H et al (2012) Hepatocellular adenoma and focal nodular hyperplasia: value of gadoxetic acidenhanced MR imaging in differential diagnosis. Radiology 262: 520-529

32. Grazioli L, Morana G, Kirchin MA, Schneider G (2005) Accurate differentiation of focal nodular hyperplasia from hepatic adenoma at gadobenatedimeglumine-enhanced MR imaging: prospective study. Radiology 236:166-177 (reviewer 4, comment 4)

33. Haimerl M, Wächtler M, Platzek I et al (2013) Added value of GdEOB-DTPA-enhanced Hepatobiliary phase MR imaging in evaluation of focal solid hepatic lesions. BMC Med Imaging 13:41 (reviewer 2, comment 24)

34. Morana G, Grazioli L, Kirchin MA et al (2011) Solid hypervascular liver lesions: accurate identification of true benign lesions on enhanced dynamic and hepatobiliary phase magnetic resonance imaging after gadobenatedimeglumine administration. Investig Radiol 46:225-239 (reviewer 4, comment 5)

35. Scharitzer M, Ba-Ssalamah A, Ringl H, Kölblinger C, Grünberger T, Weber M, Schima W (2013) Preoperative evaluation of colorectal liver metastases: comparison between gadoxetic acid-enhanced 3.0-T MRI and contrast-enhanced MDCT with histopathological correlation. Eur Radiol 23:2187-2196

36. Kim YK, Park G, Kim CS, Yu HC, Han YM (2012) Diagnostic efficacy of gadoxetic acid-enhanced MRI for the detection and characterisation of liver metastases: comparison with multidetector-row CT. Br J Radiol 85:539-547

37. Han NY, Park BJ, Sung DJ et al (2014) Chemotherapy-induced focal hepatopathy in patients with gastrointestinal malignancy: gadoxetic acid-enhanced and diffusion-weighted MR Imaging with clinical-pathologic correlation. Radiology 271:416-425

38. Péporté AR, Sommer WH, Nikolaou K, Reiser MF, Zech CJ (2013) Imaging features of intrahepatic cholangiocarcinoma in Gd-EOBDTPA-enhanced MRI. Eur J Radiol 82:101-106

39. Park HJ, Kim YK, Park MJ, Lee WJ (2013) Small intrahepatic mass-forming cholangiocarcinoma: target sign on diffusionweighted imaging for differentiation from hepatocellular carcinoma. Abdom Imaging 38:793-801

40. Verloh N, Haimerl M, Rennert J et al (2013) Impact of liver cirrhosis on liver enhancement at Gd-EOB-DTPA enhanced MRI at 3 Tesla. Eur J Radiol 82:1710-1715

41. Phongkitkarun S, Limsamutpetch K, Tannaphai P, Jatchavala J (2013) Added value of hepatobiliary phase gadoxetic acid- enhanced MRI for diagnosing hepatocellular carcinoma in highrisk patients. World J Gastroenterol 19:8357-8365

42. Haimerl M, Verloh N, Fellner C et al (2014) MRI-based estimation of liver function: Gd-EOB-DTPA-enhanced T1 relaxometry of 3T vs. the MELD score. Sci Rep 4:5621

43. Verloh N, Haimerl M, Zeman F et al (2014) Assessing liver function by liver enhancement during the hepatobiliary phase with Gd-EOB-DTPA-enhanced MRI at 3 Tesla. Eur Radiol 24:1013-1019

44. Cereser L, Furlan A, Bagatto D et al (2010) Comparison of portal venous and delayed phases of gadolinium-enhanced magnetic resonance imaging study of cirrhotic liver for the detection of contrast washout of hypervascular hepatocellular carcinoma. J Comput Assist Tomogr 34:706-711 (reviewer 4, comment 3)

45. Bartolozzi C, Battaglia V, Bargellini I et al (2013) Contrastenhanced magnetic resonance imaging of 102 nodules in cirrhosis: correlation with histological findings on explanted livers. Abdom Imaging 38:290-296

46. Yu MH, Kim JH, Yoon JH et al (2014) Small ( $\leq 1-\mathrm{cm})$ hepatocellular carcinoma: diagnostic performance and imaging features at gadoxetic acid-enhanced MR imaging. Radiology 271:748-760

47. Granito A, Galassi M, Piscaglia F et al (2013) Impact of gadoxetic acid (Gd-EOB-DTPA)-enhanced magnetic resonance on the noninvasive diagnosis of small hepatocellular carcinoma: a prospective study. Aliment Pharmacol Ther 37:355-363 (reviewer 3, comment 5)

48. Kitajima K, Yoshikawa T, Seo Y et al (2011) A case of Budd-Chiari syndrome: Gd-EOB-DTPA-enhanced MR findings. Magn Reson Imaging 29:579-583

49. Shin NY, Kim MJ, Lim JS et al (2012) Accuracy of gadoxetic acidenhanced magnetic resonance imaging for the diagnosis of sinusoidal obstruction syndrome in patients with chemotherapy-treated colorectal liver metastases. Eur Radiol 22:864-871

50. Lee NK, Kim S, Lee JW et al (2009) Biliary MR imaging with GdEOB-DTPA and its clinical applications. Radiographics 29:17071724

51. Boraschi P, Donati F (2013) Biliary-enteric anastomoses: spectrum of findings on Gd-EOB-DTPA-enhanced MR cholangiography. Abdom Imaging 38:1351-1359

52. Boraschi P, Donati F (2014) Postoperative biliary adverse events following orthotopic liver transplantation: assessment with magnetic resonance cholangiography. World J Gastroenterol 20:1108011094 